\title{
IL MAESTRO DEGLI AMBONI ZARATINI ${ }^{1}$
}

\section{IVAN JOSIPOVIĆ}

UDC: $736.2(497.581 .1) " 07 / 08 "$

Original scientific paper

Manuscript received: 12. 01. 2016.

Revised manuscript accepted: 20. 03. 2016.

DOI: 10.1484/J.HAM.5.111362
I. Josipović

Università di Zara

Dipartimento di Storia dell'arte Obala kralja Petra Krešimira IV. 2 23000 Zadar, Croatia ijosipov@unizd.hr????

The article defines a number of relief carvings found at Pridraga, Novigrad, Zadar and Nevidani on the island of Pašman. Autor identifies them as products of the same stone-cutting workshop, naming it the Master of Zadar Ambos. The carvings demonstrate features of early preRomenesque sculpture: soft modelling, loosely arranged motifs of fragile form, as well as representations of human figures which in the late eighth or the early ninth century will start to withdrow and to give primacy to an exclusively vegetative, geometrical and zoomorphic visual repertoire. Taking into account all stylistic, morphological and iconographic features of the relief carvings under consideration and the sites from which they originally come from, the author arrives at the conclusion that they belonged to the liturgical furnishings of at least two early Christian churches (Zadar - cathedral, Pridraga - St. Martin) which were renovated in the late eighth or the early ninth century.

Keywords: Master of Zadar Ambos, early pre-Romanesque sculpture, Zadar (Zara), Pridraga, Novigrad (Novegradi), Nevidane on the island of Pasman, late eighth and the early ninth century

Negli ultimi cinquant'anni lo studio della scultura altomedievale in Croazia si è concentrato sul raggruppamento di rilievi omogenei che, dal punto di vista esecutivo, sono stati riconosciuti come prodotti di un unico maestro o di una stessa bottega scultoria. In base ad alcune iscrizioni che menzionano un sovrano croato o l'anno di intitolazione della chiesa è stato possibile datare precisamente un gran numero di rilievi appartenenti a gruppi ben definiti ${ }^{2}$. Spesso il ricorso a questo metodo ha permesso anche la datazione delle chiese arricchite di questi rilievi, e perfino di interi gruppi di edifici sacri dalle caratteristiche costruttive comuni, per esempio il gruppo delle "chiese paleocroate a contrafforti arrotondati" che per la sua originalità è di una certa importanza nell'ambito del patrimonio altomedioevale europeo ${ }^{3}$.

Allo stesso modo è possibile riunire in un unico insieme alcuni rilievi con connotazioni stilistiche proprie della prima scultura preromanica ${ }^{4}$ rinvenuti a Zara (Zadar) e in altre tre vicine località dalmate: a Neviđane sull'isola di Pašman, a Pridraga e a Novegradi (Novigrad). Alcuni rilievi di questo gruppo sono stati riconosciuti come opere di un'unica produzione scultorea, ma non sono stati mai percepiti come un insieme artistico.

Già nell'anno 1896 Frano Radić pubblicò i frammenti di rilievi rinvenuti nella chiesetta di San Michele che si trova nel cimitero del villaggio di Neviđane sull'isola di Pašman. Si tratta di frammenti di plutei relativi ad un cancello presbiteriale (fig. 2) ead un parapetto d'ambone (fig. 1a)5. Dopo quasi un secolo, nel 1979 durante i lavori di ricostruzione della chiesa cimiteriale, si portarono in luce ulteriori frammenti,

\footnotetext{
${ }^{1}$ Questo articolo è la versione riadattata e parzialmente rivista, ma anche completata con minimi interventi, del capitolo omonimo della tesi di dottorato dal titolo Predromanički reljefi na teritoriju Sklavinije Hrvatske između Zrmanje i Krke do kraja 9. stoljeća (I rilievi preromanici sul territorio del ducato croato medievale tra i fiumi Zrmanja e Krka fino alla fine del IX secolo) discussa dall'autore dell'autore di questo contributo il 6 dicembre 2013 alla Facoltà di Filosofia di Zagabria.

${ }^{2}$ Si tratta di un approccio nell'analisi dei rilievi altomedioevali che ha portato al riconoscimento e alla definizione di un notevole numero (più di dieci) di opere di maestri e di botteghe attivi sulla sponda orientale dell'Adriatico dalla fine dell'VIII al principio del XII secolo, per esempio il Maestro del pluteo di Koljane, il Maestro dei capitelli di Bale, la Bottega di scultura del tempo del duca Trpimir o il cosiddetto Gruppo di Zara-Knin (bottega di scultura). Pur esistendo su questo tema una letteratura già abbastanza ampia, fino ad oggi non esiste un'opera completa che tratti integralmente tutte le unità morfologiche finora definite della scultura preromanica e primo romanica della sponda orientale dell'Adriatico. Un particolare merito per il riconoscimento e la definizione di tali manufatti va a Ivo Petricioli, Nikola Jakšić e Miljenko Jurković; tuttavia in questa sede mi limiterò a citare solo gli studi più datati di questi autori in relazione alla produzione scultorea menzionata, si veda I. PETRICIOLI, Pojava romaničke skulpture u Dalmaciji, Zagreb, 1960; N. JAKŠIĆ, Klesarstvo u službi evangelizacije: studije iz predromaničke skulpture na Jadranu, Split, 2015; M. JURKOVIĆ, Le "Maître des chapiteaux de Bale", Hortus Artium Medievalium, 8, Zagreb - Motovun, 2002, pp. 349-36o.

${ }^{3}$ I. PETRICIOLI, Oko datiranja umjetničkih spomenika ranoga srednjeg vijeka, in: Gunjačin zbornik, (ed.) Ivan Erceg et alii, Zagreb, 1980, pp. 114-117; I. PETRICIOLI, Prilog diskusiji o starohrvatskim crkvama s oblim kontraforima, Izdanja Hrvatskog arheološkog društva, 8 (Znanstveni skup “Cetinska krajina od prethistorije do dolaska Turaka", Sinj, 3-6. VI. 1980.), Split, 1984, pp. 221-226.

${ }^{4}$ Si deve a Tonči Burić una definizione sintetica, e allo stesso tempo molto informativa e precisa, dei caratteri della prima scultura preromanica a proposito della quale si dice che: "La caratterizza l'uso frequente di motivi vegetali, l'incompletezza della composizione, la rusticità della lavorazione e il rilievo accentuato rispetto alla fase successiva (preromanico maturo, N. d. A.), come anche l'uso raro dei tipici motivi a intreccio (trecce, cerchietti e quadrati a lavorazione dei nastri a tre capi e composti in serie geometriche)". T. BURIĆ, Predromanička skulptura iz Trogira, Starohrvatska prosvjeta, serie III, 12, Split, 1982, p. 156. ${ }^{5}$ F. RADIĆ, Izvješće o radu hrvatskog starinarskog družtva u Kninu u obće, a napose o kršćanskim starinama do sad odkrivenim i objelodanjenim u Dalmaciji, osjem Solina, Bosni-Hercegovini, Hrvatskoj, Slavoniji i Istri, Starohrvatska prosvjeta, serie I, II/3, Knin, 1896, pp. 179-180; F. RADIĆ, Ostaci starinske crkve i groblja u Gornjim Koljanima kod Vrlike, Starohrvatska prosvjeta, ser. I, V/3-4, Knin, 190o, pp. 107-122; F. RADIĆ, Ostanci starohrvatske crkvice S. Mihovila u Nevidjanim na otoku Pašmanu, Starohrvatska prosvjeta, ser. I, VI/3-4, Knin, 1901, pp. 84-86.
} 


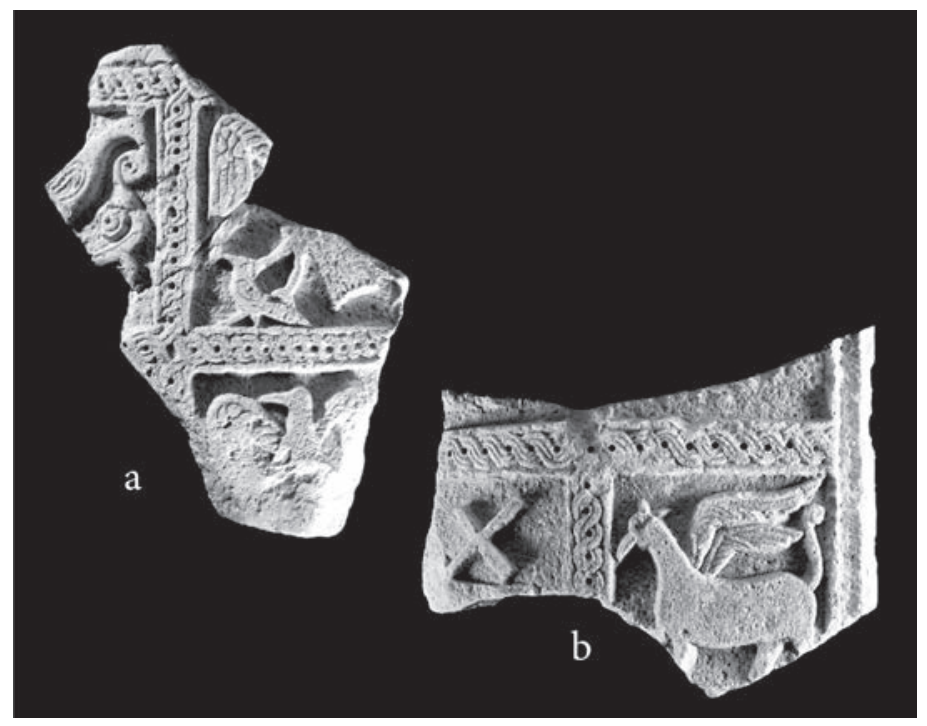

Fig. 1. Il Maestro degli amboni zaratini, Frammenti di parapetto d'ambone ritrovati a Nevidane

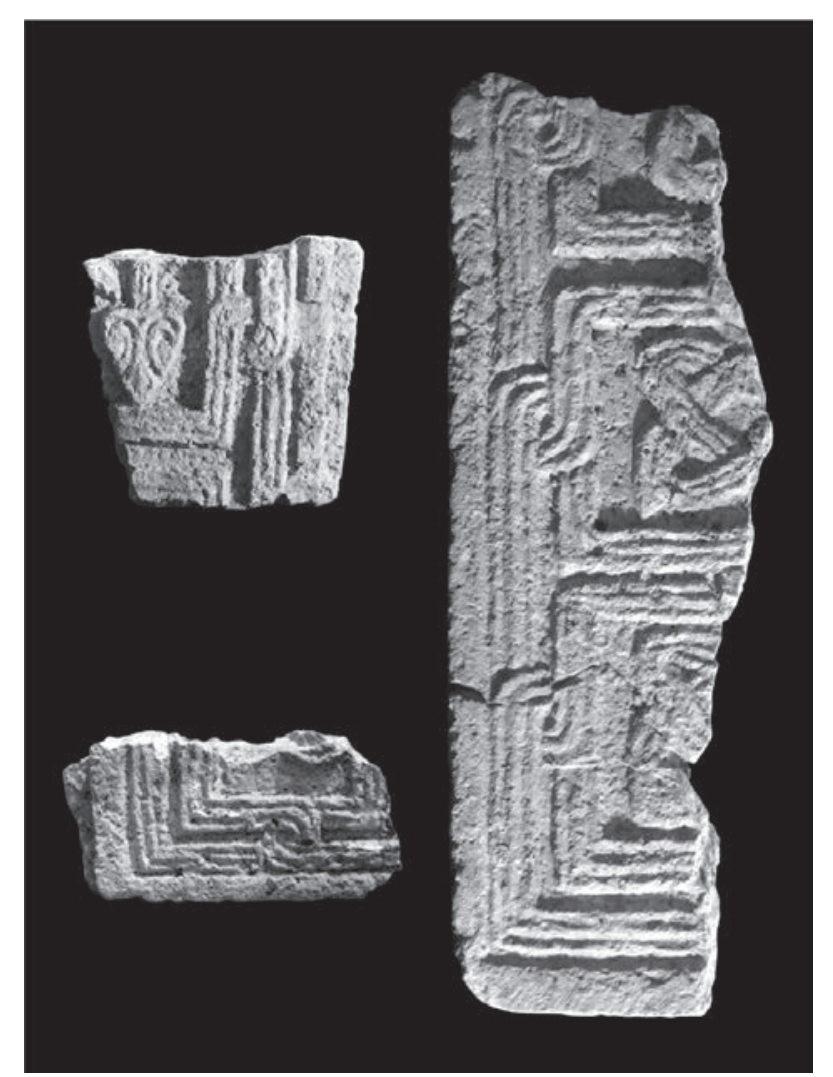

Fig. 3. Il Maestro degli amboni zaratini, Frammenti di plutei ritrovati a Nevidane nell'anno 1979

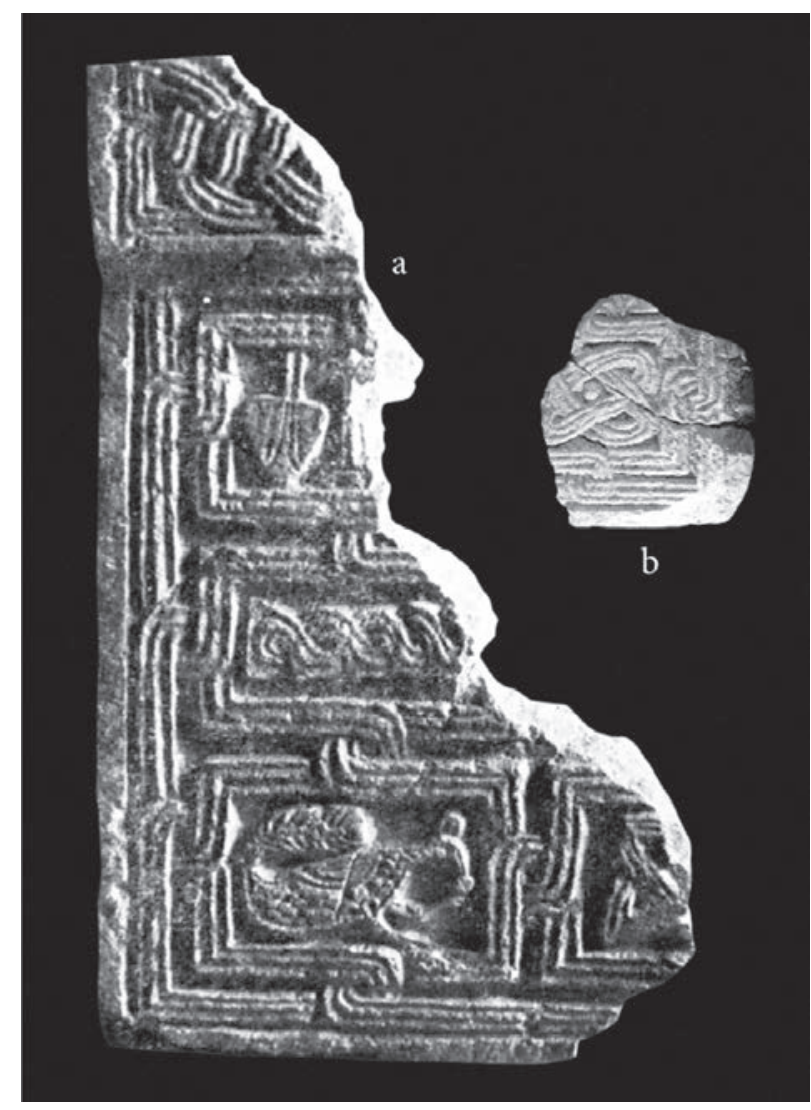

Fig. 2. Il Maestro degli amboni zaratini, Frammenti di plutei ritrovati a Nevidane nell'anno 1896

dei quali solo uno è stato pubblicato (fig. 1b) in quanto simile a quello già edito nell'articolo di Radić (fig. 1a) ${ }^{6}$, mentre gli altri non sono stati mai discussi in letteratura (fig. 3).

Successivamente, in due occasioni, Ivo Petricioli pubblicò due rilievi preromanici recuperati nella cattedrale di Zara che collegò ai frammenti d'ambone di Neviđane (fig. 1). Uno di essi faceva parte di un ambone (fig. 4), ${ }^{7}$ mentre l'altro, conservato solo come impronta nell'intonaco, faceva parte di un pluteo che rappresentava un cavaliere sotto un'arcata (fig. 5). ${ }^{8}$ A questi Petricioli aggiunse alcuni rilievi con rappresentazioni antropomorfe provenienti da Pridraga nell'entroterra zaratino (fig. 6a, 6d-e) affermando che il confronto tra i rilievi di Zara, Neviđane e Pridraga permette di avanzare questa riflessione: "that we are dealing with one workshop, probably from Zadar, which produced them in a shorter period of time" . In questa occasione Petricioli presentò l'ipotesi che anche i frammenti di Neviđane provenissero da Zara, a suo parere proprio dalla cattedrale, e propose due varianti del possibile aspetto originario dell'ambone preromanico ${ }^{10}$. Ino-

\footnotetext{
${ }^{6}$ Il frammento è stato pubblicato per la prima volta nel catalogo della mostra Sjaj zadarskih riznica tenutasi a Zagabria nel 1990. The Splendour of Zadar Treasuries. Religious Art in the Archdiocese of Zadar $4^{\text {th }}-18^{\text {th }}$ centuries (catalogue), (ed.) Tugomir Lukšić, Zagreb, 1990, pp. 308, 352, cat. n. 53.

7 Pubblicato per la prima volta in: I. PETRICIOLI, Ulomci ranosrednjovjekovnog ambona zadarske katedrale, Radovi Instituta za povijest umjetnosti, 1213/1988-1989 (Milanu Prelogu u spomen), Zagreb, 1989, pp. 25-26. Vedi anche recentemente: N. JAKŠIĆ, Riflessi della "rinascenza liutprandea" nei centri urbani della costa Adriatica orientale, Hortus Artium Medievalium, 16, Zagreb - Motovun, 2010, pp. 23-24, Fig. 18-19; N. JAKŠIĆ, La scultura "longobarda" in Croazia, in: Letteratura, arte, cultura italiana tra le due sponde dell'Adriatico (Atti della Giornata di studio, Padova, 9 novembre 2007), (ed.) Luciana Borsetto, Nedjeljka Balić-Nižić, Živko Nižić, Zadar, 2014, pp. 20-21, figg. 15-16.

${ }^{8}$ Insieme al pezzo di intonaco sono stati ritrovati anche frammenti più piccoli dello stesso rilievo in marmo che era stato riutilizzato come parte del pavimento. Il calco in gesso è stato pubblicato per la prima volta in: I. PETRICIOLI, Sculpture in Zadar between the Late Roman and pre-Romanesque Periods, Hortus Artium Medievalium, 1, Zagreb - Motovun, 1995, p. 8o, Fig. 12. Vedi, anche: N. JAKŠIĆ, op. cit. (n. 8, 2014), pp. 21-22, Fig. 18.

9 I. PETRICIOLI, op. cit. (n. 8), p. 81. Vedi, anche: I. PETRICIOLI, Predromanički ambon zadarske katedrale i srodna skulptura, in: Starohrvatska spomenička baština. Rađanje prvog hrvatskog kulturnog pejzaža, (ed.) Miljenko Jurković, Tugomir Lukšić, Zagreb, 1996, pp. 212-213.

${ }^{10}$ I. PETRICIOLI, op. cit. (n. 8), pp. 79-8o, Fig. 11; I. PETRICIOLI, op. cit. (n. 9, 1996), p. 211, Fig. 4-5.
} 


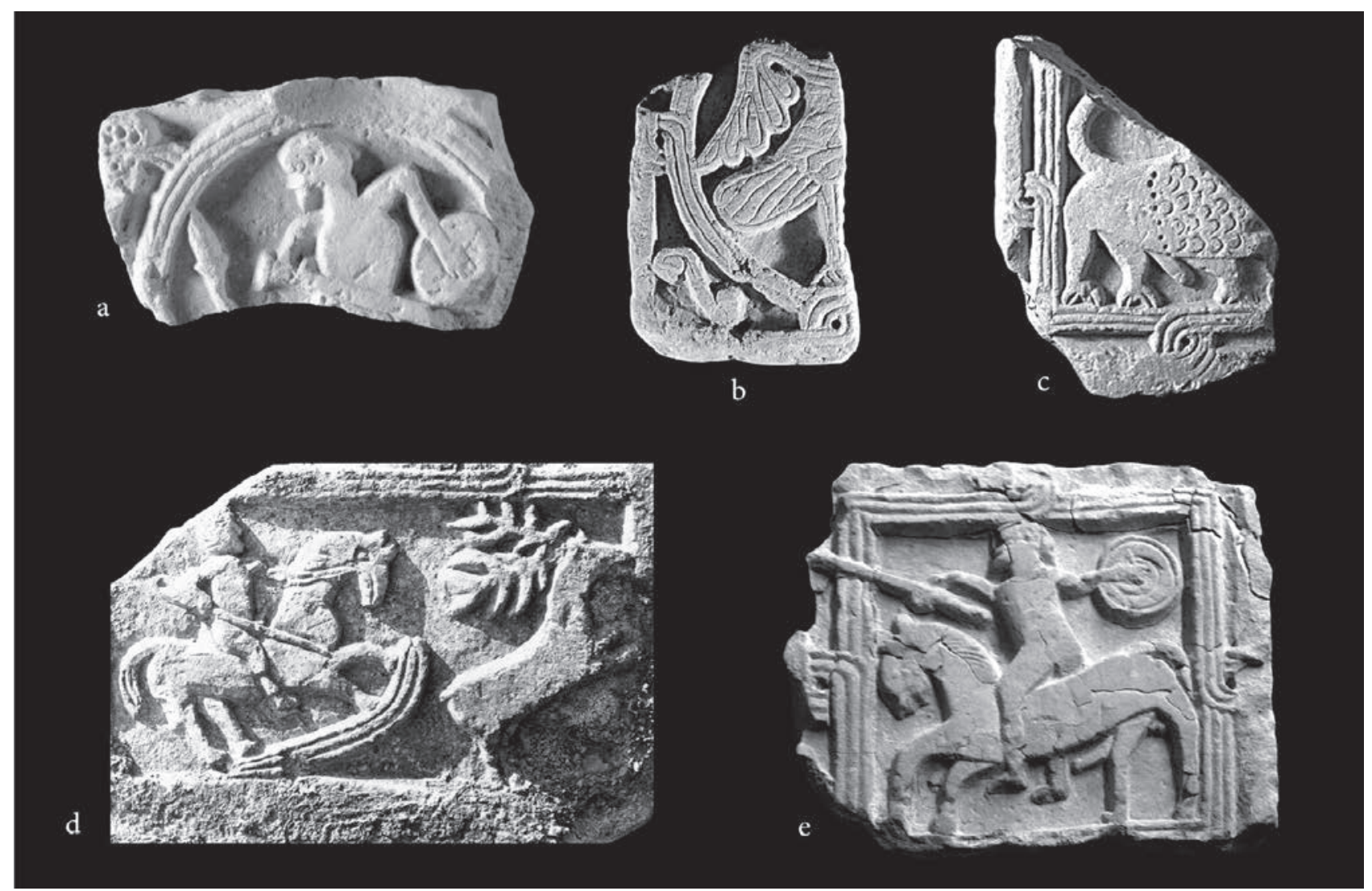

Fig 6. Il Maestro degli amboni zaratini, Frammenti di plutei con rappresentazioni antropomorfe e zoomorfe da Pridraga

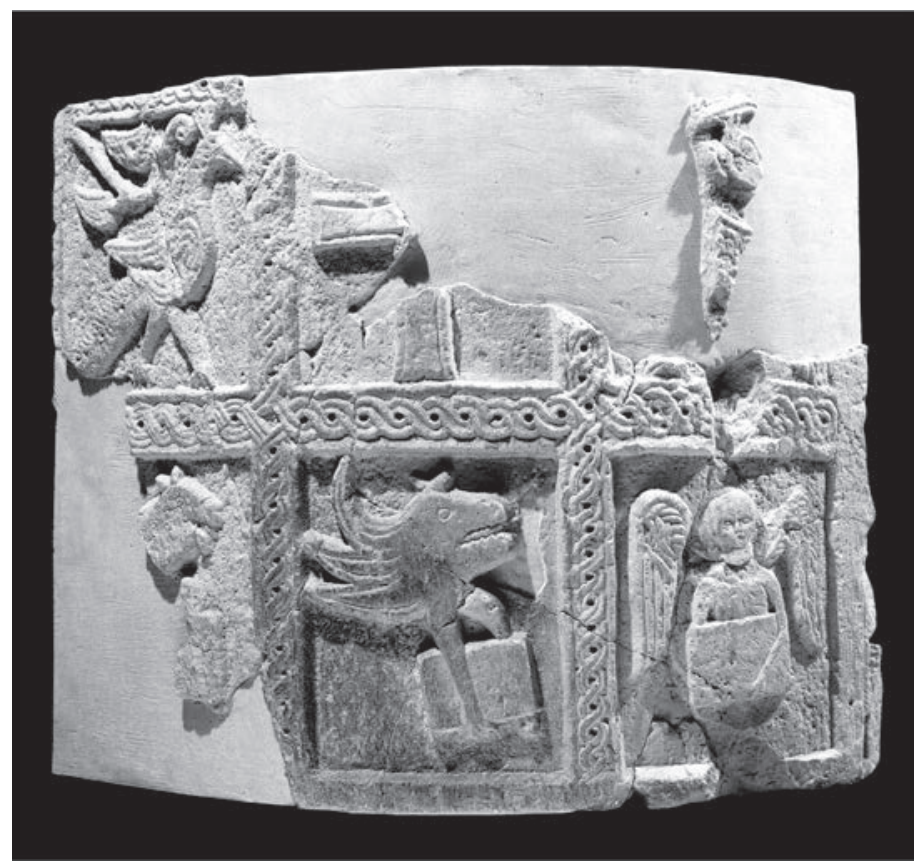

Fig. 4. Il Maestro degli amboni zaratini, Parapetto d'ambone dalla cattedrale di Zara

ltre, confrontò il parapetto d'ambone della cattedrale di Zara con la ben nota lastra del patriarca Sigualdo $(756-786 / 787)$ (fig. 13e $)^{11}$, ma anche con altre sculture risalenti alla metà e alla seconda metà dell'VIII secolo in Italia, come il pluteo di Civita Castellana (fig. 13a), le facce del sarcofago di Gussago (fig. 13d) e il rilievo della chiesa di San Saba a Roma (fig. 13c).

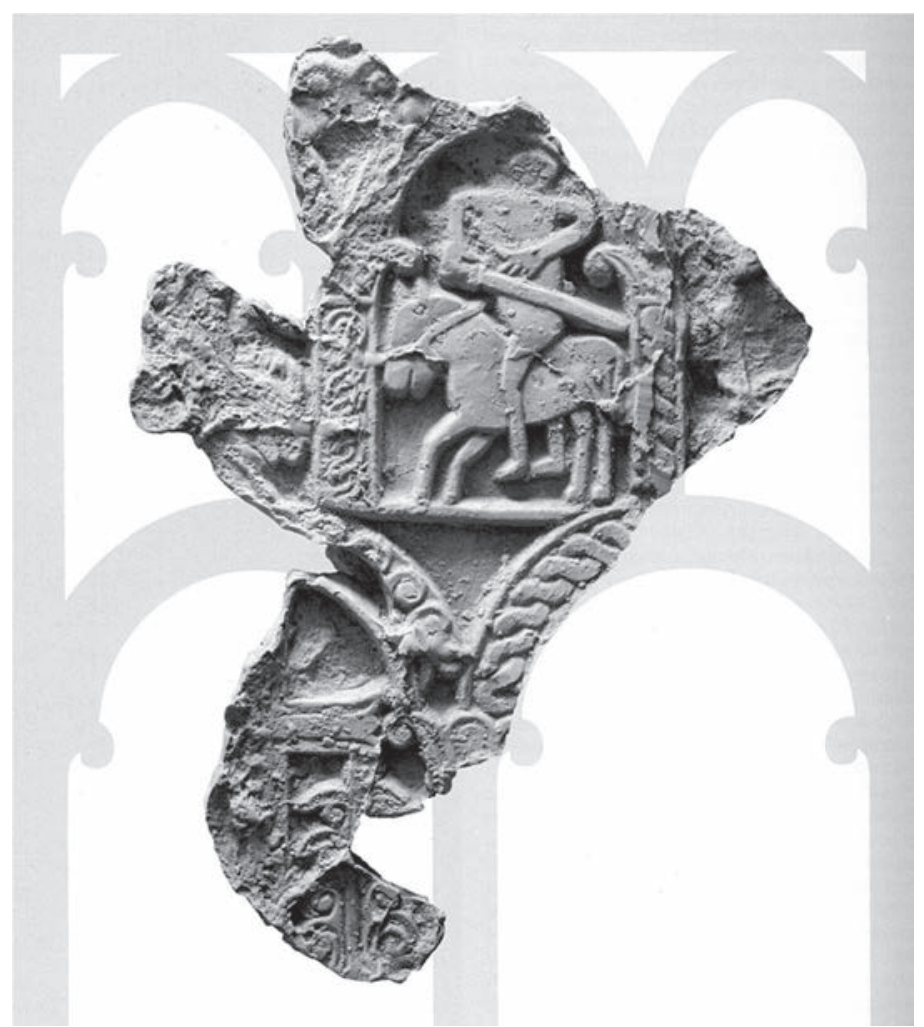

Fig. 5. Il Maestro degli amboni zaratini, Pluteo con rappresentazione di un cavaliere sotto un'arcata dalla cattedrale di Zara

Stabilendo queste analogie Petricioli concluse che in tutte queste opere è evidente lo stesso "linguaggio figurativo" e

"Petricioli come fondamentali coincidenze tra il parapetto d'ambone della cattedrale di Zara e la lastra di Sigualdo a Cividale cita la modellazione a basso rilievo e la bidimensionalità della decorazione, quindi la figurazione stilizzata, la riduzione dei corpi ai componenti fondamentali, la modellazione delle code degli animali che terminano come le volute, e la stilizzazione delle foglie. I. PETRICIOLI, op. cit. (n. 7), p. 25; I. PETRICIOLI, op. cit. (n. 8), p. 81; I. PETRICIOLI, op. cit. (n. 9, 1996), p. 213. 
perciò datò anche i rilievi di Zara e di Pridraga tra la seconda metà dell'VIII e gli inizi del IX secolo ${ }^{12}$.

D’altra parte, i frammenti di plutei preromanici di Pridraga, che sono noti in primis per le scene con figure umane (il cavaliere e il guerriero, e il rilievo recentemente rinvenuto raffigurante un cacciatore di cervi ${ }^{13}$ ), sono presenti nella letteratura storico-artistica fin dalla fine del XIX secolo, subito dopo la loro messa in luce, avvenuta intorno alla chiesa di San Martino ${ }^{14}$. Nel 1954 Kruno Prijatelj, analizzando "le sculture con figura umana di epoca paleocroata", si soffermò sui tre rilievi antropomorfi di Pridraga e, nonostante i confronti con i sopramenzionati rilievi italiani risalenti all'VIII secolo, li datò alla seconda metà o alla fine dell'XI secolo ${ }^{15}$. Su tali manufatti scrisse a più riprese anche Petricioli che, in base alle già ricordate analogie stilistiche e iconografiche, li datò alla seconda metà dell'VIII o ai primi anni del IX secolo. Per il significativo aspetto distintivo di queste opere coniò l'espressione "l'antichità nell'arte naïf altomedioevale" e li collegò alla simile scultura della cattedrale di Zara ${ }^{16}$. Alle sue osservazioni sulle caratteristiche comuni delle rappresentazioni con figure umane di Pridraga non rimane praticamente nulla da aggiungere, se non enfatizzare un dettaglio di particolare importanza relativo ai loro corpi nudi e sproporzionati che furono modellati goffamente e sembrano privi di scheletro ${ }^{17}$. Seguendo le conclusioni di Petricioli sui contesti di Zara, Neviđane e Pridraga ha scritto a più riprese anche Nikola Jakšići ${ }^{18}$, ipotizzando che i frammenti del parapetto di ambone di Neviđane proven- gano dalla chiesa di San Grisogono a Zara' ${ }^{19}$. Inoltre ritiene che entrambi i parapetti zaratini facessero parte del tipo di ambone su cui queste lastre convesse non si innalzavano dal pavimento dell'edificio, bensì dal livello dell'alto basamento eretto su colonne ${ }^{20}$, come quello nella chiesa di Sant'Apollinare Nuovo a Ravenna ${ }^{21}$. Riguardo alla datazione dell'ambone della cattedrale zaratina afferma quanto segue: "Sebbene presenti tutte le peculiarità stilistiche della scultura della metà dell'VIII secolo, è possibile concludere che tale ambone sia stato costruito in un'epoca leggermente successiva, all'inizio del IX secolo, nel periodo in cui Zara riconobbe per breve tempo il sovrano franco: proprio allora fu possibile uno stretto contatto con i forti centri religiosi sotto la dominazione franca, come Cividale o Aquileia" ${ }^{22}$. Condividendo pienamente il suo parere, Silvia Lusuardi Siena e Paola Piva hanno inoltre fatto rilevare ancora una volta la notevole somiglianza tra gli amboni zaratini (fig. 1, 4) e la lastra di Sigualdo di Cividale (fig. 13e ${ }^{23}$. Recentemente sui frammenti di Pridraga e Zara ha scritto anche Ante Milošević analizzando alcuni rilievi altomedioevali della Dalmazia centrale e della Bosnia ed Erzegovina, prima di tutto il rilievo murato sulla facciata della chiesa dell'Assunzione della Beata Vergine Maria a Žrnovnica nei pressi di Spalato ${ }^{24}$. Va ricordata la sua affermazione che l'ex pluteo marmoreo della cattedrale di Zara mostra un'espressione un po' più naïf rispetto ai rilievi di Pridraga. ${ }^{25}$ Inoltre, Milošević ha notato che il cavallo e il leone sui rilievi di Pridraga hanno il fallo eretto (figg. 6c, 6e) ed ha avanzato l'ipotesi molto intrigante che potrebbe

${ }^{12}$ I. PETRICIOLI, op. cit. (n. 7), pp. 25-26; I. PETRICIOLI, op. cit. (n. 8), pp. 81-82; I. PETRICIOLI, op. cit. (n. 9, 1996), pp. 212-214. In questo contesto ricordo che di recente Ante Milošević si è espresso sul fatto che i rilievi di Pridraga raffiguranti guerrieri e cavalieri presentano solo somiglianze generiche con gli esemplari citati della Penisola appenninica, mentre sono molto più simili ai rilievi preromanici datati verso la metà del IX secolo in Asturia. Tra essi si distingue in particolare il rilievo di cavaliere murato all'interno della chiesa di Santa Cristina de Lena (fig. 13b) datato al tempo di Ordone I (85o-866). A. MILOŠEVIĆ, Il bassorilievo altomedievale del cavaliere di Žrnovnica in Dalmazia, Godišnjak Centra za balkanološka ispitivanja Akademije nauka i umjetnosti Bosne i Hercegovine, 35, Sarajevo, 2008, p. 19o. Personalmente ritengo che questa somiglianza, peraltro giustificatissima, non cambi sostanzialmente niente rispetto alla datazione da me proposta dei frammenti di Zara e Pridraga. Si tratta cioè di una distanza geografica eccessiva che rende difficile trarre conclusioni concrete riguardo alla datazione.

${ }^{13}$ Fino a poco tempo si riteneva che il rilievo raffigurante il c.d. cacciatore di cervo fosse andato perduto. È scomparso da Novegradi nel periodo tra la Prima e Seconda guerra mondiale, dove certamente giunse dalla vicina Pridraga. Su questo rilievo e le circostanze della sua scomparsa da Novegradi vedi più dettagliatamente in: I. JOSIPOVIĆ, Predromanički reljefi na teritoriju Sklavinije Hrvatske između Zrmanje i Krke do kraja 9. stoljeća (doktorski rad, 1. dio), Zagreb, 2013, pp. 536-538, cat. n. XIII.5. Tuttavia, di recente Goran Bilogrivić lo ha riconosciuto nel frammento murato nella villa Werner, Tuškanac 36 a Zagabria. Bilogrivić ha presentato prove convincenti sul fatto che si tratti dell'originale, e non di un suo calco, ed ha esposto una spiegazione plausibile sul tempo e sulle circostanze in cui il rilievo di Novegradi giunse nel luogo dove si trova oggi. G. BILOGRIVIĆ, Ulomak pluteja s prikazom lova na jelena iz Novigrada i ljetnikovac Werner u Zagrebu, Radovi Instituta za povijest umjetnosti, 38, Zagreb, 2014, pp. 41-50.

${ }^{14}$ Vedi la letteratura critica nelle schede di catalogo in: I. JOSIPOVIĆ, op. cit. (n. 13), pp. 531-538, cat. n. XIII.1 - XIII.5.

${ }^{15}$ K. PRIJATELJ, Skulpture s ljudskim likom iz starohrvatskog doba, Starohrvatska prosvjeta, ser. III, 3, Zagreb, 1954, pp. 78-80.

${ }^{16}$ I. PETRICIOLI, Reljef konjanika iz Pridrage, Diadora, 8, Zadar, 1975, pp. 111-117; I. PETRICIOLI, Tragom srednjovjekovnih umjetnika, Zagreb, 1983, pp. 47-52; I. PETRICIOLI, op. cit. (n. 8), pp. 81-82; I. PETRICIOLI, op. cit. (n. 9, 1996), pp. 212-213.

${ }^{17}$ I. PETRICIOLI, op. cit. (n. 16, 1975), p. 113; I. PETRICIOLI, op. cit. (n. 16, 1983), pp. 48-49. Inoltre, l'autore pone in rilievo anche la modellazione delle teste con i visi rappresentati di profilo, connotati da naso aguzzo e storto e mento appuntito.

${ }^{18}$ L'ultima volta in: N. JAKŠIĆ - E. HILJE, Umjetnička baština Zadarske nadbiskupije - Kiparstvo I (od IV. do XVI. stoljeća), Zadar, 2008, pp. 26, 86-94, cat. n. o11-015, con tutta la letteratura precendente.

${ }^{19}$ Infatti, la chiesa di San Michele a Neviđane era proprietà dell'abbazia benedettina zaratina di San Grisogono fino alla sua soppressione. I. OSTOJIĆ, Benediktinci u Hrvatskoj i ostalim našim krajevima, vol. II, Benediktinci u Dalmaciji, Split, 1964, pp. 56-58; E. HILJE, Spomenici srednjovjekovnoga graditeljstva na Pašmanu, in: Toponimija otoka Pašmana, (ed.) Vladimir Skračić, Zadar, 2006, p. 67, note 47; N. JAKŠIĆ - E. HILJE, op. cit. (n. 18), p. 89.

${ }^{20}$ N. JAKŠIĆ - E. HILJE, op. cit. (n. 18), pp. 86-89.

${ }^{21}$ Vedi per esempio: L'arte in Italia, volume II, Dal secolo Val secolo XI, Da Roma ai Comuni, (ed.) Carlo Ludovico Ragghianti, Roma, 1968, Fig. 185.

${ }^{22}$ N. JAKŠIĆ, Scultura e liturgia, in: Bizantini, Croati, Carolingi. Alba e tramonto di regni e imperi, (ed.) Carlo Bertelli et alii, Milano, 2001, p. 184.

${ }^{23}$ S. LUSUARDI SIENA - P. PIVA, Da Pemmone a Paolino d'Aquileia: Appunti sull'arredo liturgico e la scultura in Friuli tra VIII e IX sec., Hortus Artium Medievalium, 8, Zagreb - Motovun, 2002, pp. 303-304.

${ }^{24}$ A. MILOŠEVIĆ, op. cit. (n. 12), pp. 181-217; A. MILOŠEVIĆ, Ranosrednjovjekovni reljef iz Maloga Čajna kod Visokog s dodanim natpisom velikog kaznaca Nespina, Godišnjak Centra za balkanološka ispitivanja Akademije nauka i umjetnosti Bosne i Hercegovine, 41, Sarajevo, 2012, pp. 187-200; A. MILOŠEVIĆ, Traces of Ancient Beliefs in Early Medieval Christianity, Dubrovnik - Split, 2013, pp. 11-87.

${ }_{25}$ Dalle sue considerazioni si può concludere che non li ritiene opera dello stesso maestro, ossia della stessa bottega di scultura, cfr. A. MILOŠEVIĆ, op. cit. (n. 12), p. 191. 


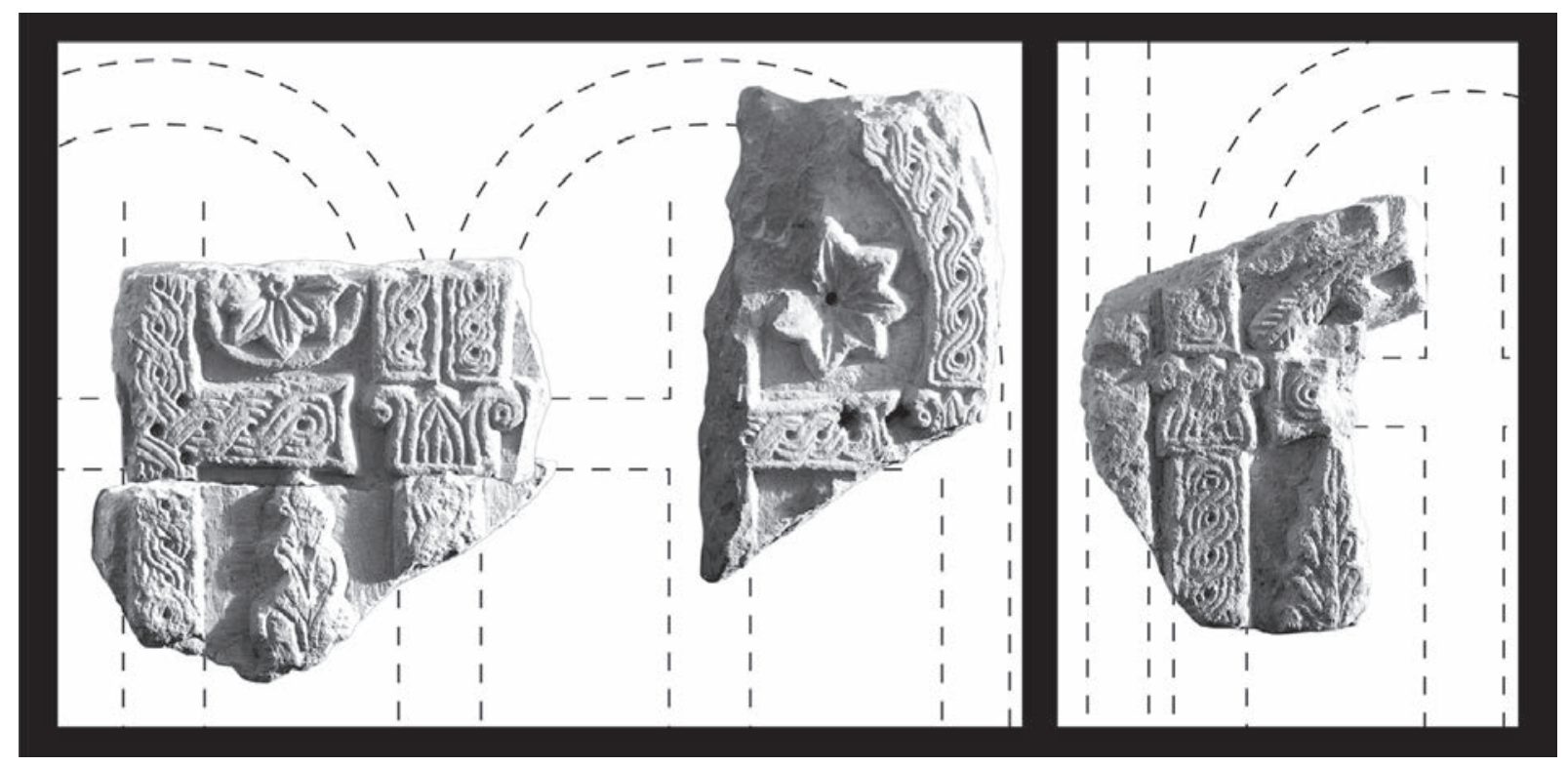

Fig. 8. Il Maestro degli amboni zaratini, Frammenti di due plutei del cancello presbiteriale da Pridraga

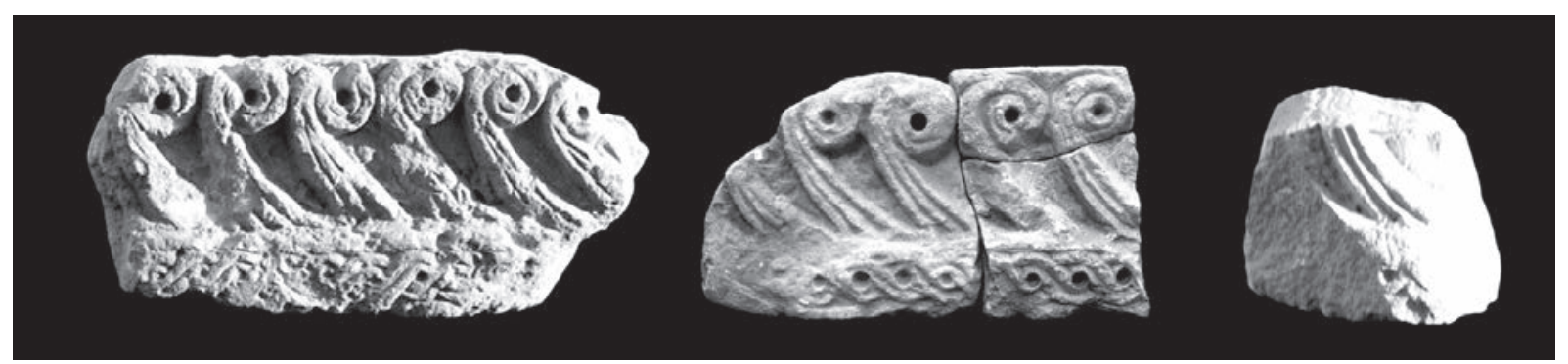

Fig. 9. Il Maestro degli amboni zaratini, Frammenti di architravi del cancello presbiteriale da Pridraga

trattarsi della sopravvivenza di antichi culti pagani (tra l'altro anche del culto dell'antica divinità della fertilità Silvano) che attraverso un particolare sincretismo iconografico sarebbero stati trasformati nell'interpretazione cristiana della rappresentazione simbolica della resurrezione ${ }^{26}$.

Petricioli, dunque, avendo collegato i rilievi di Zara, Neviđane, Pridraga e Novegradi sotto il profilo esecutivo, aveva già affermato che siamo sulla traccia di una stessa bottega scultorea ${ }^{27}$. Questa opinione potrebbe esser verificata da un'ulteriore analisi dei frammenti non ancora pubblicati di Neviđane e Pridraga ${ }^{28}$. Dopo l'analisi dell'intero corpus dei rilievi in questione parrebbe possibile definire l'operato di una produzione scultorea il cui autore potrebbe esser denominato il Maestro degli amboni zaratini.

I frammenti che si possono finora riconoscere come prodotti di tale maestro facevano parte di parapetti d'ambone, plutei, pilastri, capitelli e architravi di cancelli presbiteriali. $\mathrm{Su}$ tutti questi frammenti è evidente un aspetto distintivo legato alla maniera esecutiva del maestro. Si tratta di una modalità realizzativa riconoscibile nei rilievi incisi più profondamente che genera l'evidente effetto di chiaroscuro accentuato con l'uso di fori eseguiti a trapano, particolarmente visibile su due frammenti di capitelli del cancello di Pridraga (fig. 7). Dal punto di vista iconografico il Maestro degli amboni zaratini ha una scelta molto ampia di motivi,

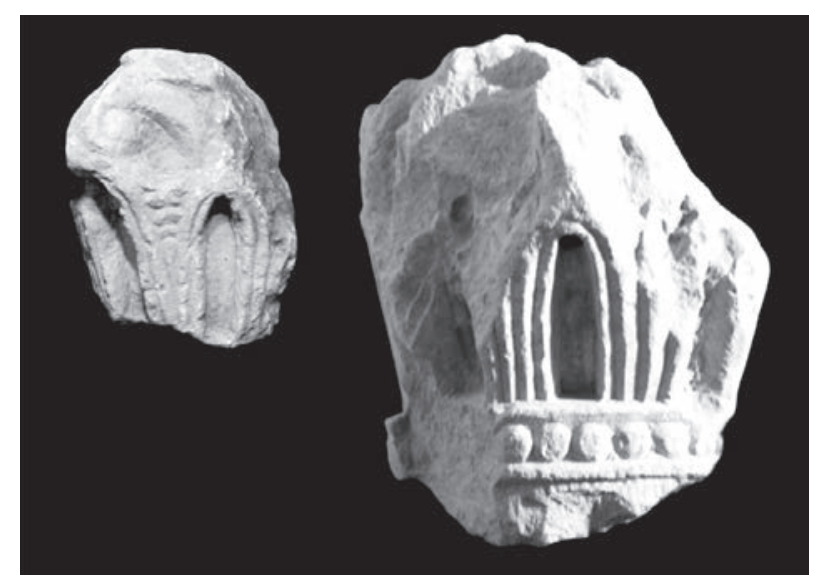

Fig. 7. Il Maestro degli amboni zaratini, Frammenti di capitelli del cancello presbiteriale da Pridraga.

prima di tutto le raffigurazioni tipiche dell'età paleocristinana (per esempio croci sulle arcate, nodi di Salomone, palmette, rosette, viticci vegetali di vario tipo ed intrecci a doppio o triplice nastro), oltre a figure zoomorfe e antropomorfe racchiuse entro rettangoli e cerchietti annodati.

I parapetti d'ambone della cattedrale di Zara (fig. 4) e quelli di Neviđane (fig. 1) mostrano la stessa forma convessa e la stessa matrice decorativa. Sono entrambi articolati mediante pannellature rettangolari e molto probabilmente organizzati in due ordini, ognuno dei quali suddiviso in tre riquadri, il che significa che originariamente ve n'erano sei su

${ }^{26}$ A. MILOŠEVIĆ, op. cit. (n. 12), pp. 75-8o, Fig. 78.

${ }^{27}$ I. PETRICIOLI, op. cit. (n. 8), p. 81; I. PETRICIOLI, op. cit. (n. 9, 1996), pp. 212-213.

${ }^{28}$ Anche a Pridraga si tratta prevalentemente di frammenti rinvenuti durante gli anni Settanta e alcuni nella seconda metà degli anni Novanta del XX secolo intorno alla chiesa di San Martino. 


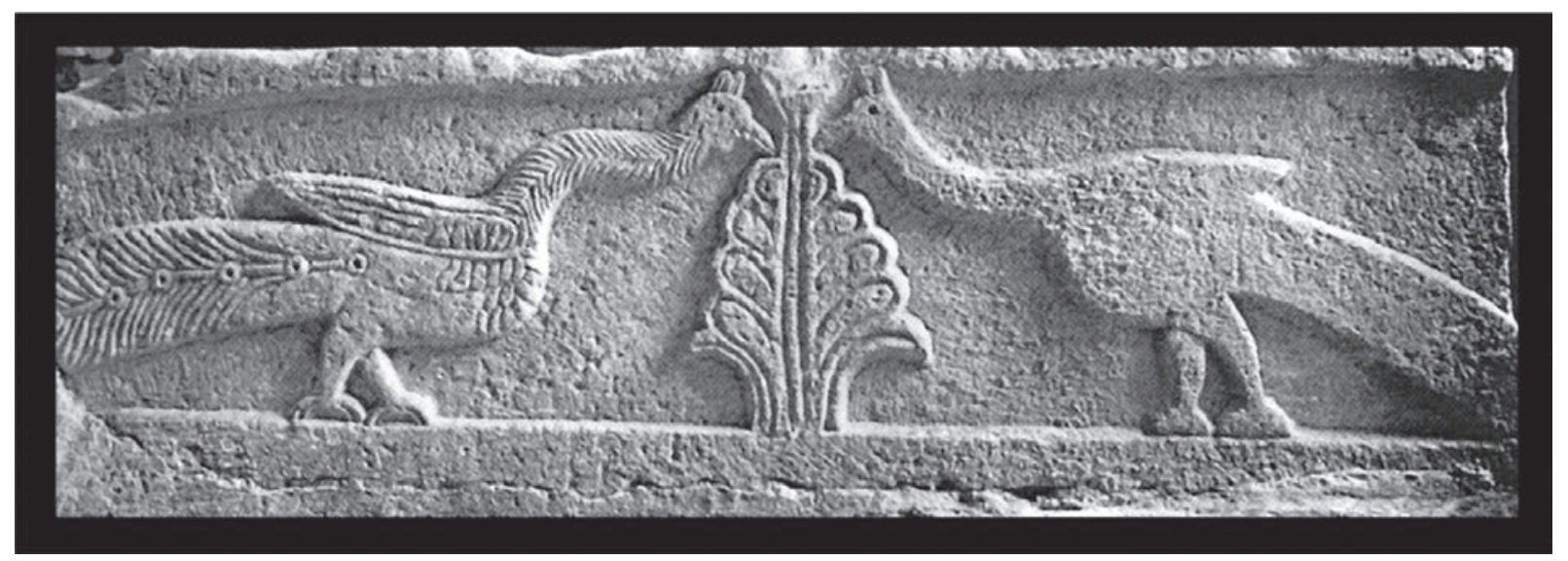

Fig. 11. Il Maestro degli amboni zaratini, Rilievo con la rappresentazione di due pavoni rinvenuto nella cattedrale di Zara

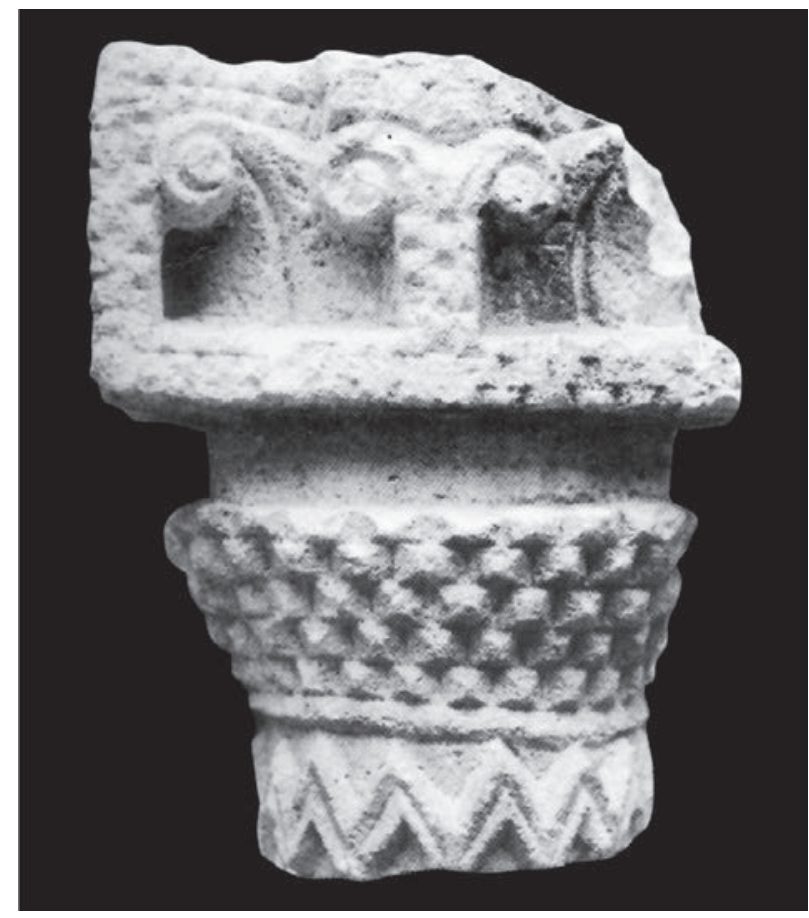

Fig. 10. Il Maestro degli amboni zaratini, Capitello proveniente dalla cattedrale di Zara

ogni lastra ${ }^{29}$. Sul frammento meglio conservato proveniente dalla cattedrale le pannellature sono bordate con intrecci di nastri a due o a tre capi arricchiti da piccoli fori eseguiti a trapano. La stessa cosa è visibile anche sul frammento relativo ad uno dei parapetti d'ambone di Neviđane (fig. 1b). Anche sui frammenti di Pridraga appaiono gli stessi intrecci con nastri a due o a tre capi (figg. 8-9). Nei riquadri presenti sull'ambone della cattedrale sono raffigurati svariati motivi, per esempio una croce greca, pavoni ed i simboli dei tre evangelisti: il leone di Marco, il vitello di Luca e l'imago hominis di Matteo, mentre nei frammenti di parapetto d'ambone di Neviđane (fig. 1) appaiono altri motivi, per esempio l'ippogrifo, un uccello predatore che ghermisce un piccolo volatile, un uccello in volo e verosimilmente la rappresentazione dell'Agnus Dei. La loro modalità esecutiva

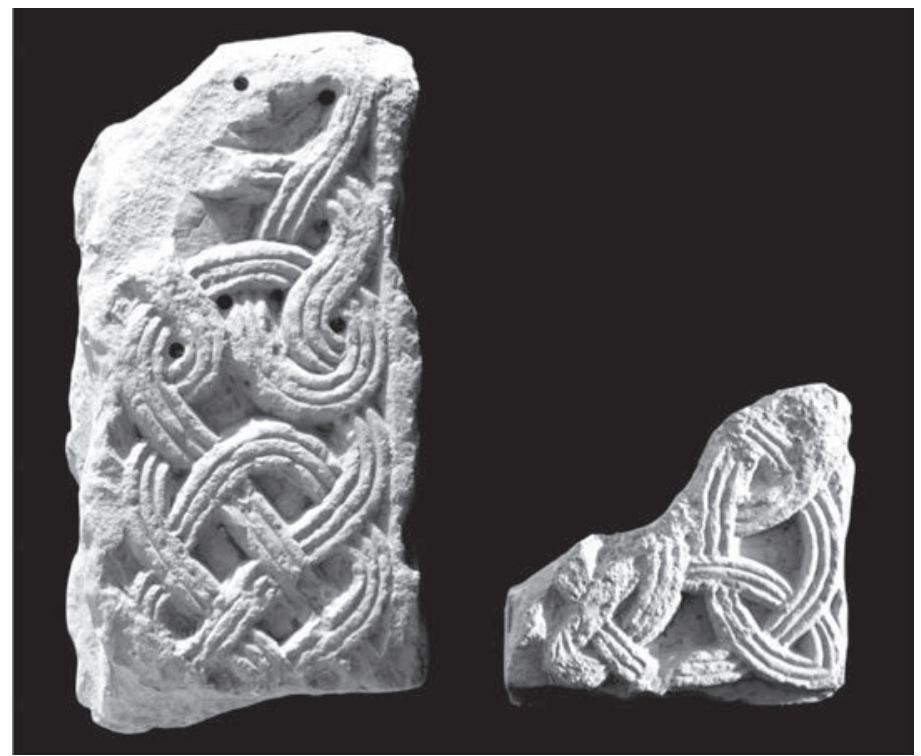

Fig. 12. Il Maestro degli amboni zaratini, Frammenti di pilastri del cancello presbiteriale da Pridraga

è molto specifica, in quanto nei loro corpi stilizzati e prevalentemente lisci si notano alcune parti trattate con una certa accuratezza. La stessa modalità esecutiva è visibile sui frammenti di plutei raffiguranti guerrieri (fig. 6a), cavalieri (fig. 6d-e) e quadrupedi (fig. 6c) che appartenevano alla chiesa di San Martino a Pridraga. Queste raffigurazioni sono collocate entro cerchietti, quadrati e rettangoli annodati a triplice nastro ugualmente arricchito da forellini eseguiti a trapano. Proprio la suddivisione in pannelli quadrati e rettangolari che racchiudono diversi motivi figurativi e decorativi collega a livello concettuale i plutei di Pridraga con i frammenti ritrovati a Neviđane (figg. 2-3). Accanto ai soliti motivi vegetali egeometrici su un pluteo frammentario di Neviđane appare anche un senmurv, motivo insolito nei rilievi in Dalmazia (fig. 2a).

Tutti i frammenti di Zara e Pridraga mostrano un'evidente somiglianza per l'espressione naïf e per la similarità iconografica con il pluteo zaratino conosciuto dal calco in gesso (fig. 5$)^{30}$. Sebbene ci siano delle differenze evidenti nella

\footnotetext{
${ }^{29}$ In questa occasione convengo con la proposta di ricostruzione dei parapetti degli amboni presentata da Nikola Jakšić che si differenzia da entrambe le varianti offerte da Ivo Petricioli (i parapetti con quattro o cinque ordini di cassette in altezza). La proposta di Jakšić si fonda sull'opinione che si tratti dei frammenti di amboni di due chiese zaratine (la cattedrale e San Grisogono (?)), cioè che i loro parapetti appartenessero ad amboni innalzati su colonne. N. JAKŠIĆ - E. HILJE, op. cit. (n. 18), pp. 26, 86-89.

$3^{30}$ Riguardo a quanto esposto sopra, Ante Milošević ha presentato un parere completamente diverso e per questo si veda: A. MILOŠEVIĆ, op. cit. (n. 12), p. 191. In ogni caso, Nikola Jakšić ha avvertito (similmente a Ivo Petricioli prima di lui) che certi particolari del pluteo zaratino, per esempio la modellazione del guerriero, presentano caratteristiche simili ai rilievi di guerriero e di cavaliere a Pridraga. N. JAKŠIĆ - E. HILJE, op. cit. (n. 18), pp. 92-94, cat. n. o15, Fig. o15.
} 


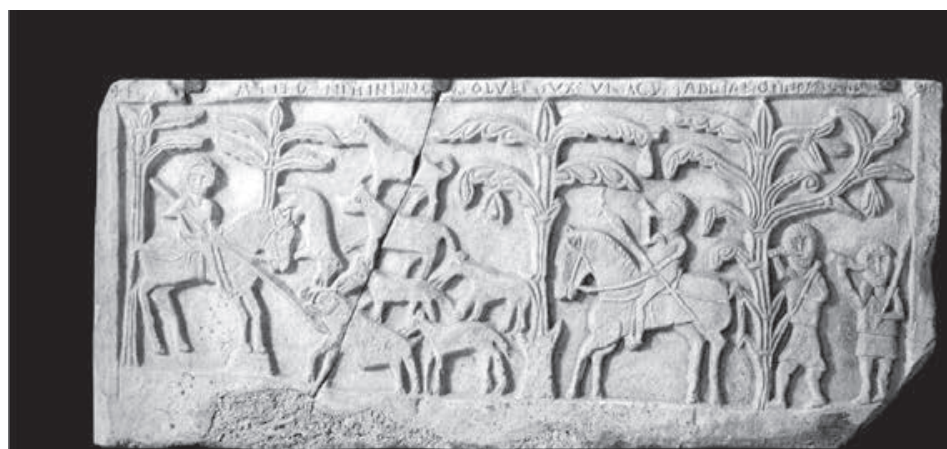

a) Civita Castellana

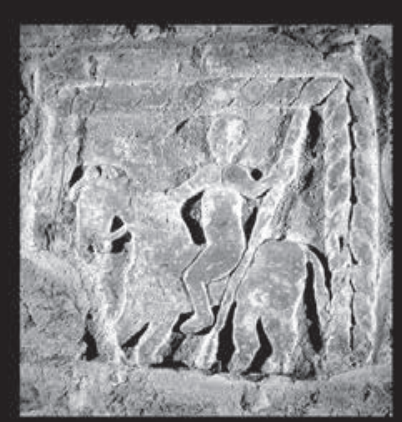

b) Santa Crisitina de Lena

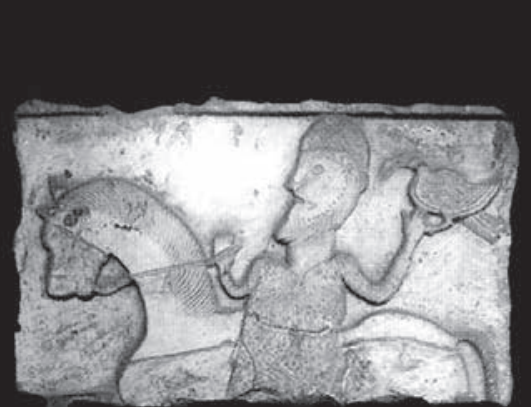

c) Rim, San Saba

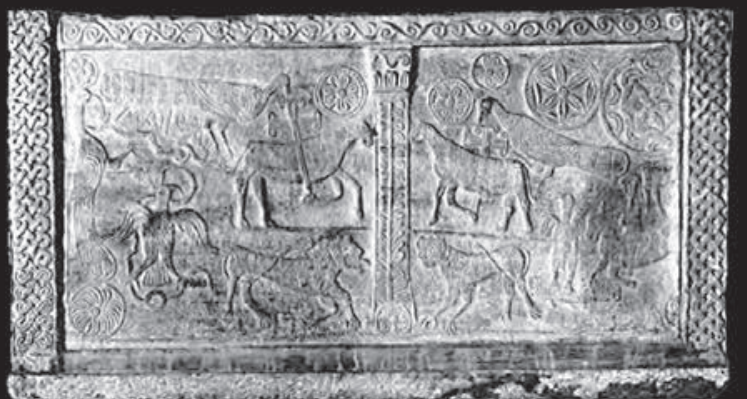

d) Gussago

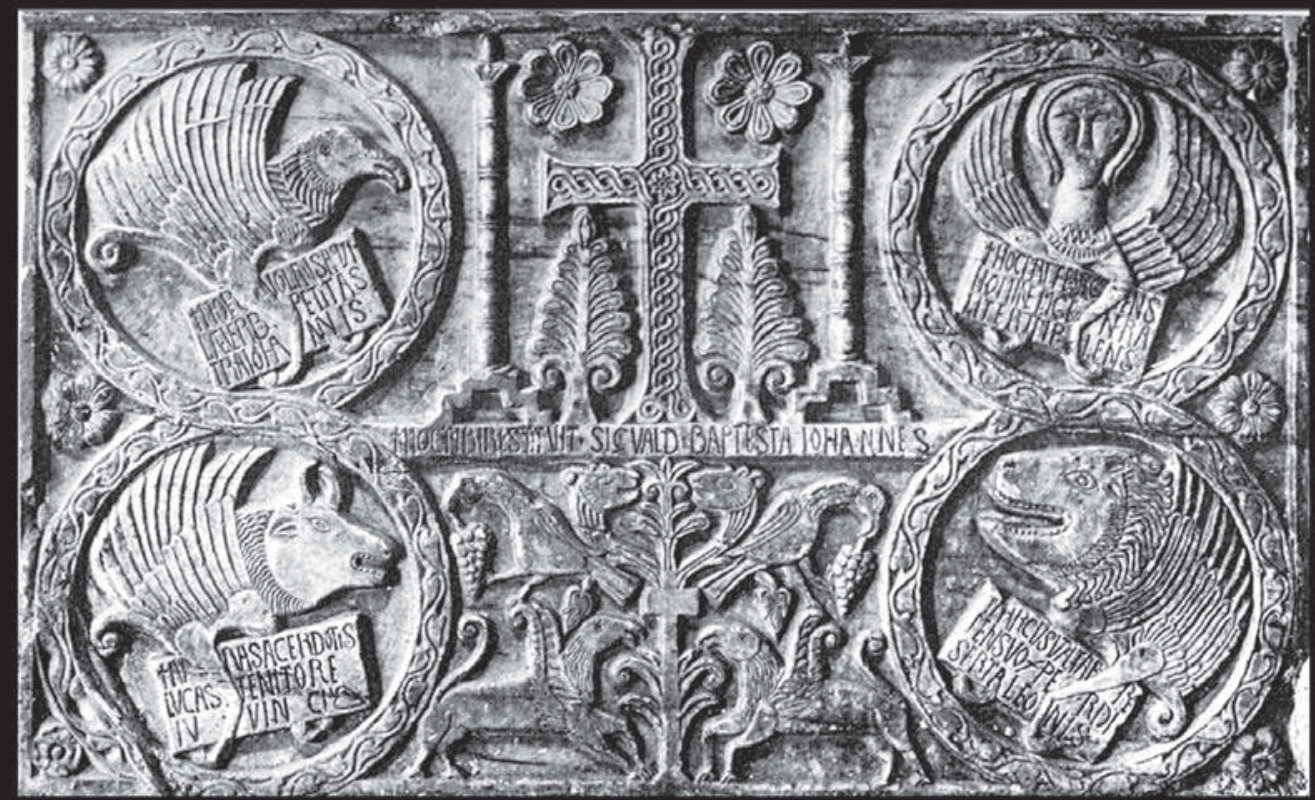

e) Cividale

Fig. 13. Cinque rilievi preromanici di diversa funzione dall'talia e dalla Spagna

modellazione e nell'aspetto generale, si possono notare molti dettagli esecutivi comuni. Alcuni particolari di questo pluteo (piccole volute) si vedono anche su un capitello proveniente dalla cattedrale di Zara (fig. 10) ${ }^{31}$. Con il Maestro degli amboni zaratini si può porre in relazione un altro rilievo rinvenuto nella cattedrale di Zara raffigurante due pavoni contrapposti che beccano l'Albero della vita (fig. 11) ${ }^{32}$. Anche su questo rilievo tutti i particolari anatomici di un pavone vengono elaborati dettagliatamente, mentre il corpo dell'altro è del tutto liscio, caratteristica già rilevata nei rilievi di questa produzione a Zara e Pridraga. D’altra parte, i già citati pavoni di Zara ricordano un pavone scolpito su una delle arcate del

${ }^{31}$ Pavuša Vežić ritiene che si tratti del capitello di un ciborio della cattedrale o della rotonda di Santa Trinità (San Donato) datandolo alla seconda metà dell'VIII secolo. P. VEŽIĆ - M. LONČAR, HOC TIGMEN - Ciboriji ranoga srednjeg vijeka na tlu Istre i Dalmacije, Zadar, 2009, pp. 78-79.

${ }^{32}$ I. PETRICIOLI, op. cit. (n. 8), p. 78, Fig. 7. 
ciborio del vescovo Maurizio a Cittanova d'Istria (Novigrad istarski) datato all'ultimo quarto dell'VIII secolo ${ }^{33}$.

Tranne i rilievi con figure umane e animali entro cerchietti, quadrati e rettangoli annodati, il Maestro degli amboni zaratini a Pridraga scolpiva anche plutei con motivi completamente diversi. A questo proposito, infatti, si segnalano tre frammenti di plutei raffiguranti croci entro una teoria di arcate (fig. 8) ${ }^{34}$. Su tali manufatti si notano le caratteristiche tipiche di questo maestro già rilevate in precedenza: uso dell'intreccio a due e a tre capi, fori eseguiti a trapano e plasticità dei motivi floreali che conferiscono alla composizione un effetto chiaroscurale. Le stesse caratteristiche morfologiche sono presenti su alcuni frammenti di pilastri, capitelli e architravi (figg. 7, 9, 12).

Per concludere si può ricordare che si tratta evidentemente di un maestro dotato di una certa inventiva che, a colpi "tremanti" di scalpello, eseguiva motivi figurati vari di forme esili. Tra questi spiccano in particolare le rappresentazioni antropomorfe che, proprio a quel tempo, cominciavano decisamente a cedere il posto a soggetti vegetali e geometrici che sarebbero diventati la caratteristica dello stile preromanico maturo. Le solide analogie stilistiche stabilite tra le opere del Maestro degli amboni zaratini e alcuni rilievi noti della penisola appenninica dell'VIII secolo ne suggeriscono una datazione abbastanza corrispondente, cioè la fine dell'VIII o l'inizio del IX secolo. Allo stesso modo la distribuzione decorativa di alcuni plutei nel sistema di rettangoli annodati rappresenta una reminiscenza dei mosaici pavimentali paleocristiani. Inoltre, l'attività del nostro maestro è stata riconosciuta a Zara e Pridraga, nelle località che dopo l'anno 812 (pace di Aquisgrana) facevano parte di due entità politiche, rispettivamente Zara nella Dalmazia bizantina e Pridraga nel ducato croato altomediovale. Parrebbe dunque che tutti questi rilievi siano stati realizzati prima di quella data, cioè al tempo in cui Zara e Pridraga appartenevano ad un'unica diocesi ecclesiastica, ovvero il vescovato di Zara. A tale proposito, infatti, vale la pena di ricordare che i rilievi di Pridraga decoravano l'interno di un edificio sacro paleocristiano, ma non l'edificio costruito durante il IX secolo nel territorio del ducato croato. Per questo bisognerebbe considerare le origini delle opere del nostro maestro nel più ampio contesto della presenza del governo bizantino nell'entroterra delle città dalmate durante l'"epoca buia" del VII e VIII secolo35.

Traduzione dal croato: Nicoletta Russotti-Babić

\footnotetext{
33 M. JURKOVIĆ, Il ciborio di Novigrad (Cittanova d’Istria), Hortus Artium Medievalium, 1, Zagreb - Motovun, 1995, pp. 141-149, Fig. 5; M. JURKOVIĆ - I. MATEJČIĆ - J. ZIHERL, Lapidario di Cittanova, Novigrad - Cittanova, 2006, pp. 46, 53.

34 P. VEŽIĆ, Ninska crkva u ranom srednjem vijeku - problem kontinuiteta i rezultati arheoloških istraživanja, in: Starohrvatska spomenička baština. Rađanje prvog hrvatskog kulturnog pejzaža, (ed.) Miljenko Jurković, Tugomir Lukšić, Zagreb, 1996, pp. 94-95, Fig. 18.

35 N. JAKŠIĆ, Il ruolo delle antiche chiese rurali nella formazione del ducato croato medievale, Hortus Artium Medievalium, 14, Zagreb - Motovun, 20o8, pp. 103-112.
} 